\title{
SISTEMATIZACIÓN DE UN PROCESO DE FORMACIÓN DE PROMOTORES COMUNITARIOS: REFLEXIONES Y LECCIONES APRENDIDAS SOBRE LA PARTICIPACIÓN Y EL VOLUNTARIADO
}

\author{
Systematization of a training process of community promoters: \\ thoughts and learned lessons on participation and volunteer
}

David Tarazona Cervantes ${ }^{1}$, Stephanie Jerí de Pinho, Luis Bellido Canchaya

Centro de Promoción e Investigación para el Desarrollo Sostenible, Perú

(Recibido el 07/02/2011 - Aceptado el 16/06/2011)

\begin{abstract}
RESUMEN
El presente artículo presenta el proceso de formación de promotores en un programa de promoción de bibliotecas comunitarias. El trabajo de sistematización consiste en el reordenamiento crítico de los sucesos ocurridos en una experiencia para comprender las razones que determinaron que ésta se desarrolle en una forma específica y no de alguna otra forma. Se reconstruyen dos procesos, primero, el ciclo general del proyecto durante el periodo en el que se desarrolla la experiencia y luego, en segundo lugar, se presenta el desarrollo específico del proceso de formación de promotores comunitarios. Se finaliza presentando las lecciones aprendidas identificadas y desarrolladas.
\end{abstract}

Palabras clave: Voluntariado, participación, formación de promotores, psicología comunitaria, sistematización de experiencias.

\begin{abstract}
This article discusses the training of promoters in a program to promote community libraries. Systematization is a critical rearrangement of the events into an experience to understand the reasons that it develops in a specific way and not otherwise. Two processes are reconstructed first, the overall project cycle during which develops the experience and then, secondly, we present the development of process specific training of community. It ends by presenting the lessons learned identified and developed.
\end{abstract}

Keywords: Volunteer, participation, training process, community psychology, systematization of experiences. 


\section{INTRODUCCIÓN}

El Perú es una nación que desde hace ya varios años tiene a la educación como uno de sus problemas centrales. Diversas evaluaciones del rendimiento alcanzado por estudiantes de escuelas públicas dan cuenta del estado crítico que se vive en estos centros. Por ejemplo, el año 2003, la Organización para la Cooperación y el Desarrollo Económico (OCDE) y la UNESCO dieron a conocer los resultados de las evaluaciones hechas por el Programa Internacional de Evaluación de Estudiantes (Programme for International Student Assessment, PISA), cuya finalidad es conocer la medida en que los jóvenes de 15 años que se aproximan al fin de la escolaridad obligatoria están preparados para satisfacer los desafíos de las sociedades de hoy, evaluándose aptitudes en tres áreas: lectura, matemática y ciencias. El Perú obtuvo el puntaje más bajo en la escala de aptitud para la lectura, a 219 puntos del puntaje más alto (Finlandia), estando el 54 por ciento de los adolescentes en el primer nivel de dominio, lo que significa que, en el mejor de los casos, sólo pueden manejar las tareas más básicas de lectura. En cuanto al rendimiento en matemática, nuevamente el Perú obtuvo el promedio de puntuación más bajo. Esta evaluación también dio cuenta que en nuestro país se correlacionaba el bajo nivel educativo con la pobre inversión educativa.

El bajo nivel de logro alcanzado por la educación peruana fue nuevamente documentado por el Ministerio de Educación (MINEDU), que en el año 2005 publicó los resultados de la Evaluación Nacional, llevada a cabo el año anterior, dando a conocer los niveles alcanzados por estudiantes de $3 .^{\text {er }}$ y $5 .^{\text {to }}$ de secundaria, además de los de $2 .^{\text {do }}$ y $6 .^{\text {to }}$ de primaria. Entre los estudiantes de educación secundaria se halló que en comunicación sólo el 15.1 y el 9.8 por ciento de los estudiantes de $3 .^{\text {er }}$ y $5 .{ }^{\text {to }}$ años, respectivamente, alcanzaron un nivel suficiente, es decir, que lograron los aprendizajes requeridos en su nivel educativo, mientras que el 34.3 y el 30.1 por ciento, de $3 .^{\text {er }}$ y $5 .{ }^{\text {to }}$ respectivamente, sólo lograron alcanzar un nivel por debajo del previo. En el área de matemática, el panorama mostró menor desarrollo; mientras que el 6 por ciento de estudiantes de $3^{\circ}$ de secundaria alcanzó un nivel suficiente, el 55.1 por ciento sólo alcanzaron un nivel por debajo del previo; por su parte, entre los estudiantes de $5 .{ }^{\text {to }}$ de secundaria, el nivel suficiente sólo fue alcanzado por el 2.9 por ciento, contra un 68.5 por ciento que mostró un nivel por debajo del previo. Ambos estudios (PISA y la Evaluación Nacional del MINEDU) dan cuenta de la urgencia de promover habilidades básicas entre niños, niñas y adolescentes con miras a incrementar el capital humano del país.

Para afrontar esta situación se han emprendido diferentes experiencias tanto al interior del sector educación como en espacios comunitarios. En esta última alternativa se encontraba el Programa de Bibliotecas Comunales de la Municipalidad Metropolitana de Lima. Esta experiencia buscó implementar bibliotecas en zonas de alto riesgo de la ciudad que funcionaran como espacios en donde niños, niñas, adolescentes y jóvenes puedan acceder a material educativo para mejorar su aprendizaje y educación. El proyecto ponía en práctica las estrategias de generación de infraestructura pública y promoción de la participación comunitaria. La generación de infraestructura pública consistía en la implementación de bibliotecas equipadas de fácil acceso para pobladores de zonas pobres de la ciudad. La promoción de la participación comunitaria se definía por la acción organizada de promotores comunales en tareas como acopio de libros, gestión de los centros y desarrollo de actividades de animación. 
La concepción metodológica del proyecto se basó en el empoderamiento (Figura 1), es decir, buscaba dar a los pobladores beneficiarios del proyecto un rol activo en la toma de decisiones sobre las actividades y, por consiguiente, en su implementación [Agencia Suiza para el Desarrollo y la Cooperación (COSUDE), 2000, 2002; Sánchez, 2002]. De esta manera, la intervención contemplaba dos actores: el equipo del proyecto y los promotores comunales.

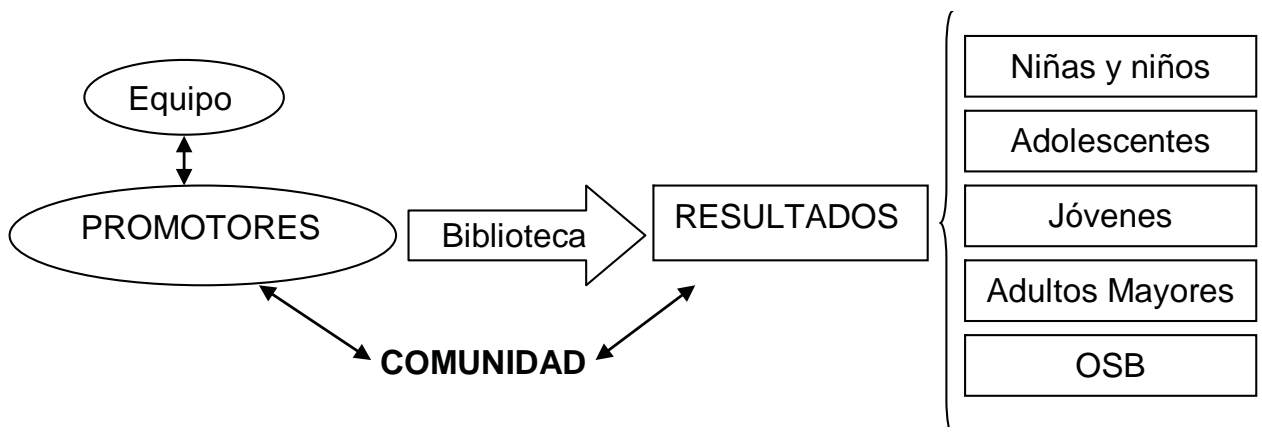

FIGURA 1. Concepción metodológica del proyecto.

La propuesta consideró al equipo de promotores comunitarios actor protagónico para el logro de los objetivos mientras el equipo del proyecto cumpliría más bien un papel facilitador. La solución de los problemas sociales respondería al trabajo de la misma comunidad por lo que esta cumpliría dos roles: beneficiaria y benefactora. Beneficiaria al obtener mayores oportunidades educativas mediante la biblioteca comunal. Benefactora porque los miembros de la misma comunidad protagonizarían la gestión de este espacio educativo. Los resultados del proyecto tendrían impacto sobre niñas y niños, adolescentes y jóvenes y adultos mayores, grupos vulnerables que requieren soporte para alcanzar un mejor bienestar y consolidar sus capacidades; pero además se logrará un impacto en las organizaciones sociales de base, espacios que agrupan a líderes comunales y cumplen en la vida comunitaria funciones relevantes de orden social, productivo e incluso de supervivencia, como por ejemplo clubes de madres, organizaciones juveniles, comités de vaso de leche y comedores populares, entre otros (Loli \& López, 1999).

Para que los pobladores de las comunidades participantes pudieran estar en capacidad de asumir roles relevantes en la implementación del proyecto, se determinó la necesidad de efectuar entre noviembre de 2003 y noviembre de 2004 el diseño e implementación de un proceso de formación de promotores comunales, incluyendo la validación de un módulo educativo que garantizara la eficacia de las actividades. El presente reporte de sistematización ${ }^{2}$ tuvo como objeto de análisis esa experiencia educativa específica (no la totalidad del proyecto) y su propósito fue formular lecciones aprendidas útiles para la sostenibilidad de las bibliotecas comunales.

2 El formato y los criterios de investigación se basan en Banister, Burman, Parker, Taylor \& Tindall (1994), en tanto las pautas referidas a la sistematización de experiencias corresponden a Jara (1994) y Barnechea, González \& Morgan (1998). 


\section{MÉTODO}

\section{Participantes}

Se involucró a todos los pobladores protagonistas de la experiencia, 28 promotores y el equipo del proyecto (4 personas).

TABLA 1. Edad y sexo de los participantes

\begin{tabular}{cccccccc}
\hline \multirow{2}{*}{ EDADES } & \multicolumn{4}{c}{ SEXO } & \multicolumn{2}{c}{ TOTAL } \\
\cline { 2 - 5 } & \multicolumn{2}{c}{ Varones } & \multicolumn{2}{c}{ Mujeres } & & \\
\cline { 2 - 6 } & $\mathrm{f}$ & $\%$ & $\mathrm{f}$ & $\%$ & $\mathrm{f}$ & $\%$ \\
\hline 14 o menos & 1 & 6.7 & 1 & 7.7 & 2 & 7.1 \\
$15-16$ & 8 & 53.3 & 4 & 30.8 & 12 & 42.9 \\
$17-18$ & 4 & 26.7 & 7 & 53.8 & 11 & 39.3 \\
19 o más & 2 & 13.3 & 1 & 7.7 & 3 & 10.7 \\
\hline TOTAL & 15 & 100.0 & 13 & 100.0 & 28 & 100.0 \\
\hline
\end{tabular}

Como puede observarse en la Tabla 1, entre los promotores se apreció un número relativamente equitativo de varones y mujeres (15 contra 13); pero las mujeres mayormente tenían 17 o 18 años (53.8 por ciento) mientras que entre los varones las edades más frecuentes fueron 15 y 16 años (53.3 por ciento). Datos adicionales indican que el 92.9 por ciento se encontraba estudiando y que el 64.3 por ciento trabajaba, asimismo que el 100 por ciento estudiaba o había egresado de colegios públicos.

\section{Instrumentos y materiales}

TABLA 2. Fuentes de información

\begin{tabular}{lcc}
\hline Registro & Objetivo & Indicadores de calidad \\
\hline Registro de reuniones & $\begin{array}{c}\text { Registrar el avance del } \\
\text { trabajo programado }\end{array}$ & $\begin{array}{c}\text { Se recopiló apuntes tomados por todos los } \\
\text { integrantes del equipo, asimismo las actas } \\
\text { de reunión que se hicieron. }\end{array}$ \\
\hline Notas de campo & $\begin{array}{c}\text { Registrar libremente } \\
\text { observaciones sobre el } \\
\text { proceso de avance }\end{array}$ & $\begin{array}{c}\text { Se tomaron durante el momento en } \\
\text { que ocurrían los sucesos recogiéndose } \\
\text { la perspectiva de más de uno de los } \\
\text { involucrados. }\end{array}$ \\
\hline
\end{tabular}




\section{Procedimientos}

El trabajo de sistematización consiste principalmente en el reordenamiento crítico de los sucesos ocurridos en una experiencia para comprender las razones que determinaron que ésta se desarrolle en una forma específica y no de alguna otra forma (Jara, 1994). En nuestro caso, además del testimonio del equipo del proyecto, la sistematización recurrió a dos fuentes principales de información, por un lado los registros y las notas de campo, mientras que en otro lado se tuvo a una evaluación hecha al final del proceso de formación de promotores.

A lo largo de toda la experiencia se emplearon registros anecdóticos (notas de campo), guardándose las anotaciones en una libreta de apuntes, sin generarse archivos electrónicos. Los testimonios de los participantes y algunos trabajos grupales realizados en los talleres si fueron 'vaciados' en archivos electrónicos de texto (*.txt) para ser analizados con el programa Atlas-Ti v.4.2. En ambos casos se hizo un análisis de contenido abierto.

Una vez organizada toda la información se procedió a la identificación de lecciones aprendidas, elaborándose una versión preliminar del reporte que fue expuesto en un evento académico. Posteriormente se ampliaron las referencias y se procedió a la redacción del presente artículo.

\section{RESULTADOS}

Los resultados se presentan buscando seguir el orden estándar de una sistematización según la propuesta de Jara (1994). Así, primero se da a conocer el proceso seguido durante la experiencia comunitaria que ha sido analizada: la formación de promotores. Luego se presentan las lecciones aprendidas en cada etapa de la experiencia. Finalmente, se informan los resultados de la evaluación de recursos psicológicos.

\section{El ciclo del proyecto}

Un proyecto social busca responder a necesidades concretas poniendo en práctica medios originales y creativos (Pérez, 1999). Debido a esto es usual que antes de la implementación de un proyecto se produzca un proceso alternado de formulación, evaluación y reformulación que permita garantizar la eficacia de las estrategias y actividades que se contemplen en el diseño de intervención. 


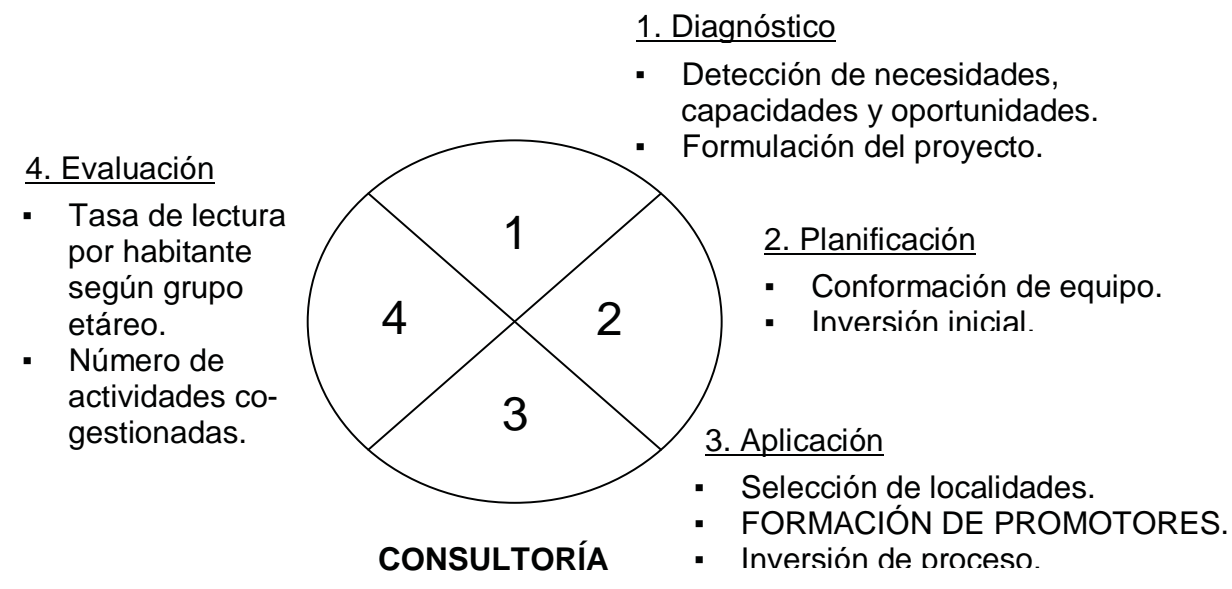

FIGURA 2. Ciclo del proyecto.

Como se aprecia en la Figura 2, el ciclo del Programa de Bibliotecas Comunales tuvo cuatro etapas. El caso de formación de promotores comunitarios analizado en el presente reporte está ubicado en la tercera etapa del ciclo del proyecto, estando precedida por acciones de diagnóstico y planificación.

\section{Diagnóstico}

Esta etapa correspondió a la detección de necesidades, capacidades y oportunidades existentes para poder concretar la formulación del proyecto. La estrategia principal seleccionada para promover el hábito de lectura fue la implementación de bibliotecas de fácil acceso a los vecinos; por ello se seleccionaron comunidades que contaran con una proporción significativa de población infantil y adolescente matriculada en centros escolares, de modo que la biblioteca pueda servir como un espacio facilitador del aprendizaje y no como un ente que duplicará funciones con las bibliotecas escolares de la zona. Otra oportunidad considerada fue la existencia de grupos organizados en la comunidad que pudieran compartir la administración de la biblioteca; de este modo se buscaría emular experiencias participativas exitosas ocurridas en zonas urbano-marginales similares a las que se incorporarían al presente proyecto.

Entre las experiencias exitosas de administración compartida que el equipo identificó como antecedentes se tienen, por un lado, a los Comités Locales de Administración de Salud, propuesta promovida por el Ministerio de Salud que juega un rol importante en el acercamiento de la población a los establecimientos de salud y en la vigilancia epidemiológica (particularmente en salud materno-infantil), además de la promoción de hábitos de higiene (Altobelli, 2002), y también, por otro lado, a los Comités de Saneamiento Ambiental, instancias que en trabajo conjunto con los gobiernos locales (municipios) han contribuido decisivamente en la gestión de residuos sólidos y la promoción del reciclado como alternativa laboral para familias de sectores pobres (Lozano \& Palacios, 2005). 


\section{Planificación}

Esta segunda etapa implicó la conformación del equipo del proyecto y la inversión inicial. Para esto ya se había aprobado el perfil presentado a partir del diagnóstico y, por consiguiente, se tenía elaborada una planificación operativa que permitió efectuar la inversión inicial, consistente en el pago al personal del proyecto y la compra de mueblería y patrimonio bibliográfico para el funcionamiento básico de las bibliotecas, adicionalmente se realizaron pequeñas convocatorias para contar con vecinos interesados en participar voluntariamente en el proyecto, dándoseles el nombre de 'promotores'. Esta situación generó una reflexión amplia en el equipo, decidiéndose mantener a los vecinos voluntarios dado su interés y compromiso pero al mismo tiempo reorientar la labor de los consultores hacia la implementación de un proceso de formación de promotores que proveyera a los voluntarios de las capacidades requeridas para efectuar su labor con mayor eficacia, además de proyectarse a lograr la administración compartida en el mediano plazo.

Así, el proyecto contaría con dos profesionales a tiempo completo (abogado y socióloga) y dos consultores a tiempo parcial (psicólogo y bibliotecaria). El rol de los consultores se concentró en la formación de los promotores, cuestión fundamental para lograr a mediano plazo la administración compartida, en tanto los profesionales a tiempo completo se responsabilizaban de labores propias de la gestión social (monitoreo, coordinación, visitas de campo, asesoría, entre otras). El rol de sistematización (incluyendo la redacción y presentación de avances) fue cumplido por el psicólogo consultor.

\section{Implementación}

La tercera etapa, aplicación o implementación del proyecto, se vio reconfigurada en tres componentes: la selección de localidades, la formación de promotores y la inversión. Si bien ya se había hecho una inversión inicial en algunas comunidades, aún no era definitiva la selección de localidades debido a que esta selección inicial respondió más a demandas políticas que al proceso técnico que conlleva la gestión del proyecto. Ante esto, se acordó continuar trabajando con las comunidades ya involucradas, que en buena cuenta se mostraban favorables al funcionamiento de las bibliotecas, y continuar la selección de nuevas comunidades basándose en los criterios referidos en el diagnóstico. La inversión contemplaba el pago al equipo, subvenciones para los participantes en casos especiales y el proceso de formación de promotores, asimismo se contempló inversión para la compra de más mobiliario y más patrimonio bibliográfico para las bibliotecas.

\section{Evaluación}

En la cuarta etapa se contempla la evaluación de dos indicadores clave: la tasa de lectura por habitante según grupo etáreo y el número de actividades co-gestionadas. Sin embargo, esta evaluación final del proyecto no se tomó en cuenta debido a un cambio de prioridades en la gestión municipal del periodo 2003 - 2006. 


\section{Proceso seguido durante la formación de promotores}

Como se señaló anteriormente, la formación de promotores (el objeto del presente reporte de sistematización) tuvo lugar en la tercera etapa del proyecto. Esta actividad educativa se entrelazaba con el trabajo de seleccionar nuevas comunidades para el proyecto, de modo que se iban desarrollando talleres formativos en forma paralela con cada comunidad involucrada, aunque en dos oportunidades se trabajó integrando a los promotores de más de una comunidad.

La experiencia de formación de promotores tuvo tres etapas: planificación, capacitación y acompañamiento (Figura 3).

\begin{tabular}{|c|c|c|c|}
\hline \multirow[b]{2}{*}{ Etapas } & $\begin{array}{c}\text { Primera } \\
\text { PLANIFICACIÓN }\end{array}$ & $\begin{array}{c}\text { Segunda } \\
\text { CAPACITACIÓN }\end{array}$ & $\begin{array}{c}\text { Tercera } \\
\text { ACOMPAÑAMIENTO }\end{array}$ \\
\hline & & & \\
\hline Proyecto & \multicolumn{3}{|c|}{ Implementación } \\
\hline $\begin{array}{l}\text { Actividades } \\
\text { principales }\end{array}$ & $\begin{array}{l}\text { - Convocatoria. } \\
\text { - Reuniones. }\end{array}$ & $\begin{array}{ll}\text { - } & \text { Talleres. } \\
\text { - } & \text { Charlas. } \\
\text { - } & \text { Reuniones. }\end{array}$ & - Reuniones. \\
\hline Resultados & $\begin{array}{l}\text { - } \\
\text { - } \\
\text { - } \\
\text { Rerfilil del de promón de } \\
\text { participantes en la } \\
\text { capacitación. }\end{array}$ & $\begin{array}{ll}\text { - } & \text { Módulo educativo } \\
\text { diseñado. } \\
\text { - } \\
\text { Talleres } \\
\text { implementados. } \\
\text { - } \text { Relación de } \\
\text { participantes } \\
\text { capacitados. }\end{array}$ & $\begin{array}{l}\text { Encuesta a } \\
\text { promotores. } \\
\text { Relación de } \\
\text { promotores. }\end{array}$ \\
\hline
\end{tabular}

FIGURA 3. Proceso seguido durante la formación de promotores.

\section{Etapa de planificación}

La primera etapa dentro del proceso de formación de promotores comunitarios fue la planificación. Al planificar una actividad se pretende "precisar los resultados a obtener y el papel que en ellos representan los elementos personales y materiales [del proyecto]" (Pérez, 1999; p. 52); en este sentido, se definió que la responsabilidad de este proceso educativo recayera en el psicólogo y la bibliotecaria dado que poseían mayor experiencia en el diseño y la gestión de capacitaciones y que venían apoyando a los promotores inicialmente incorporados. Las tareas asumidas a partir de este momento hasta el inicio de las capacitaciones tuvieron dos orientaciones, por un lado iniciar la convocatoria a los interesados, y por otro lado el diseño del modulo educativo.

Para iniciar la convocatoria a interesados se definieron como requisitos (1) la participación y el interés en formar parte del equipo promotor de la biblioteca; además de (2) estar interesado en el desarrollo de la comunidad. 
Con estos dos criterios de participación definidos se procedió a celebrar reuniones con organizaciones sociales de las comunidades (comedores, juntas vecinales, comités de vaso de leche, organizaciones juveniles) para dar a conocer el inicio de las actividades y obtener su apoyo en la distribución de volantes entre sus asociados y los vecinos. Inicialmente, esta alianza con las organizaciones sociales fue exitosa en todas las comunidades, pero conforme el proyecto se fue desarrollando ocurrieron problemas en algunas localidades debido a la divergencia de intereses, por lo cual se tuvo que renegociar algunos acuerdos y en otros casos recurrir a nuevas alianzas.

En el diseño del módulo educativo se consideró que el proceso sea de carácter formativo, es decir, que involucre no sólo la impartición de enseñanza para la consolidación de habilidades sino que además genere en los participantes una identidad con la función a desempeñar, es decir que adquieran el estatus de promotores de la biblioteca. En este sentido, el enfoque educativo asumido fue el constructivista, caracterizado por promover aprendizajes nuevos a partir de las experiencias de los participantes en un escenario de ‘interacción retroactiva' entre docentes y alumnos (González, 1998).

Las funciones propuestas a los promotores en su perfil contemplaron la gestión integral de las bibliotecas (Tabla 4), asimismo este perfil sirvió como base para la formulación de objetivos del módulo educativo (Tabla 5). Así, el primer objetivo tiene que ver con el dominio conceptual de las funciones de una biblioteca y las funciones específicas que cada promotor debe asumir; en tanto el segundo objetivo aborda las habilidades requeridas para el cumplimiento de funciones y responsabilidades de cada promotor.

TABLA 4. Perfil del Promotor Comunal

Organiza y administra la biblioteca comunal.

Atiende y asesora al público usuario.

Participa en el mantenimiento de los muebles, ambientes y patrimonio bibliográfico de la biblioteca comunal.

Planifica, coordina y ejecuta acciones de promoción de la lectura dentro de su comunidad. Colabora en la realización de actividades educativas, recreativas y en la promoción de estilos de vida saludable. 
TABLA 5. Objetivos, unidades y temas del módulo de capacitación

\begin{tabular}{|c|c|c|}
\hline Objetivos & Unidades & Temas \\
\hline \multirow{5}{*}{$\begin{array}{l}\text { Al finalizar el módulo, los partici- } \\
\text { pantes conocerán la naturaleza y el } \\
\text { funcionamiento de una Biblioteca } \\
\text { Comunal identificando sus funciones } \\
\text { y responsabilidades como Promoto- } \\
\text { res. }\end{array}$} & & Derechos culturales \\
\hline & La Biblioteca Comunal & Participación vecinal \\
\hline & & Biblioteca comunal \\
\hline & Liderazgo y trabajo en & Liderazgo / Emprendimiento \\
\hline & & Trabajo en equipo \\
\hline
\end{tabular}

Roles y procedimientos

Gestión eficiente y

Incorporarán herramientas de trabajo para la implementación y desarrollo de actividades propias de una Biblioteca Comunal en el marco de sus funciones y responsabilidades. servicio a la comunidad

Difusión de servicios

Atención al usuario

Cuidado de locales y mobiliario

Inventariado

Procesos técnicos bibliotecarios
Catalogación

Mantenimiento de libros

El número de vecinos interesados en ser promotores fue diverso en cada comunidad, pero en todos los casos superaba las 20 personas. Un punto importante a resaltar es que se buscó que en cada biblioteca se distribuya las tareas según las aptitudes y destrezas de cada miembro del equipo de promotores. De esta forma, si bien todos conocerían todas las funciones, su responsabilidad estaría delimitada periódicamente sólo a un grupo de éstas para que nadie se recargue de trabajo y puedan mantener estable su motivación (Lara, 2001).

La planificación también conlleva la previsión de estrategias correctivas (Pérez, 1999), por lo que se consideró que si bien 20 era un buen número para iniciar el trabajo de capacitación, probablemente habría casos de deserción que afecten el normal desarrollo de los talleres, ante esto se decidió que se reuniría a los grupos que estuvieran más próximos geográficamente, o bien a todos en el local municipal ubicado en el centro de la ciudad, subvencionando la participación para evitar molestias por el traslado y la pérdida de tiempo laborable 3 .

A pesar de los esfuerzos, no se pudo incorporar muchos adultos al proceso de capacitación. Mayormente los interesados que culminaron la experiencia fueron jóvenes y adolescentes, sin embargo ellos mantuvieron constantemente un vínculo con organizaciones que presentaban un 'rostro adulto', de modo que el espacio de las bibliotecas sirvió inesperadamente como una plataforma para la integración de los jóvenes a la vida comunitaria en modo análogo a como funcionan las experiencias de voluntariado social promovidas en organizaciones juveniles (Ver Tarazona, 2004). Este hecho otorgó un valor agregado a la experiencia y orientó muchas de las pautas metodológicas posteriores.

3 En caso no hubiera vecinos interesados en ser capacitados se previó que se buscaría otra comunidad interesada, situación que no se presentó. 


\section{Etapa de capacitación}

La segunda etapa correspondió a las actividades de capacitación, que es un componente metodológico importante para todo proyecto, ya que permite a sus protagonistas la adquisición de conocimientos y habilidades que representan la 'semilla' del cambio social que propugna todo proyecto de desarrollo (Bobadilla, Del Águila \& Morgan, 1998; Pérez, 1999). En nuestro caso, estos aprendizajes se orientaron a permitir que los promotores cumplan con sus funciones en aras del éxito de la biblioteca y con ello de los objetivos del proyecto; es decir, el aprendizaje estuvo centrado en que los promotores aplicaran directamente sus nuevos aprendizajes en su propio trabajo de promoción (Frings, Gachuchi, Matiru \& Müller, 1993). En total hubo cuatro procesos formativos paralelos, dos en la zona este de la ciudad, uno en la zona sur y otro en la zona norte.

Como se señaló anteriormente, el enfoque educativo asumido fue el constructivista, por lo que las actividades fueron participativas y vivenciales. El componente participativo hace que la capacitación resulte progresiva, variada y directa, con lo cual se logra "motivar, estimular y activar el descubrimiento de los hechos por uno mismo desde su propia visión del mundo, confrontándola con la visión de los demás” (Lara, 2001; p. 56-57). Para el componente vivencial se emplearon actividades artísticas que "favorecen la expresión y que constituyen formas de iniciación o de desarrollo de los lenguajes creativos y de la capacidad de innovación y búsqueda de nuevas formas expresivas" (Pérez, 1999; p. 74) asimismo se recurrió a eventos sociales que "favorecen la vida asociativa y la atención a necesidades grupales y la solución de problemas colectivos” (Pérez, 1999; p. 76).

Los participantes en la capacitación eran invitados a asumir inmediatamente algún rol en la biblioteca, buscando que la capacitación y el involucramiento en el proyecto sean paralelos. Esta medida tuvo éxito al ser las mismas bibliotecas (específicamente las salas de lecturas) el lugar donde se realizaban los talleres. Esto permitió que todos de alguna forma tuvieran un marco referencial común y pudieran hacer propuestas de aplicación directa a la realidad. Por ejemplo, al tratarse sobre la limpieza y mantenimiento de la biblioteca, el módulo proponía efectuar un concurso de afiches informativos entre los grupos conformados al interior de cada equipo de promotores recurriendo a diversos materiales para su diseño (cartulinas, cartones, telas, pinturas, plumones, papeles de diverso tipo, entre otros), ocurriendo que los afiches creados por los grupos quedaron de una vez instalados. Los trabajos alcanzaron un buen nivel de calidad y fueron muy creativos, en gran parte porque los participantes podían visualizar su utilidad directa.

Durante esta etapa se procedió a la implementación de lo planificado por lo que la mayor atención estuvo concentrada en la ejecución de talleres. En todos los casos la facilitación de las sesiones corrió a cuenta del equipo del proyecto pero en ocasiones participaba algún invitado en reemplazo de algún miembro que no tuviera disponibilidad en el día fijado. Cada proceso duró entre tres y cuatro meses y la duración de las sesiones varió sustancialmente entre dos y seis horas según las posibilidades de los participantes en cada uno de los cuatro procesos. Aunque esta variedad demandó más tiempo y recursos, permitió que cada comunidad cumpla con recibir todos los contenidos del módulo educativo que se había diseñado, lo cual es un beneficio que retribuye a largo plazo la inversión y el esfuerzo hechos. 
TABLA 6. Esquema de una sesión

\begin{tabular}{lll}
\hline \multicolumn{1}{c}{ Pasos } & \multicolumn{1}{c}{ Resultados } & \multicolumn{1}{c}{ Actividades } \\
\hline Motivar y sensibilizar & $\begin{array}{l}\text { Los participantes... } \\
\text { Indagan sobre el tema y los objetivos de la sesión. } \\
\text { Se ponen en disposición de aprender. }\end{array}$ & \\
Recoger saberes previos & $\begin{array}{l}\text { Expresan sus conocimientos y experiencias pre- } \\
\text { vias en torno al tema. }\end{array}$ & \\
Brindar información nueva & $\begin{array}{l}\text { Recogen información nueva y la integran a sus co- } \\
\text { nocimientos previos. }\end{array}$ & Según módulo \\
Practicar lo aprendido & $\begin{array}{l}\text { Aplican correctamente los aprendizajes en ejerci- } \\
\text { cios prácticos. }\end{array}$ & \\
Sintetizar y recordar ideas & $\begin{array}{l}\text { Visualizan el conjunto de aprendizajes y recuerdan } \\
\text { lo más importante. }\end{array}$ & \\
Evaluar la actividad & Expresan su opinión sobre la actividad realizada. & \\
\hline
\end{tabular}

Nota: Secuencia basada en Asociación Kallpa, Con la salud sí se juega.

En el desarrollo de cada sesión se siguieron seis etapas o pasos en busca de una relación causal entre actividades y resultados (Tabla 6). Esta secuencia metodológica evidencia el enfoque constructivista y se orientó a promover un aprendizaje activo y social, es decir, por un lado que el aprendizaje esté basado tanto en la aplicación y la experiencia como en la especulación y el error, y por otro lado, que el aprendizaje sea fortalecido por la interacción entre pares (Frings, Gachuchi, Matiru \& Müller, 1993). En relación con lo último, podría decirse que los talleres buscaban ser 'laboratorios de ensayo' protagonizados por los participantes.

Adicionalmente a los talleres se tuvieron reuniones y se dieron charlas, estas últimas principalmente a iniciativa de los mismos participantes y como forma de continuar el proceso de acercamiento a las organizaciones sociales para motivar a un mayor número de vecinos a participar en las actividades de la biblioteca. Por ejemplo, en una comunidad hubo dos jóvenes miembros de una iglesia evangélica que organizaron quincenalmente durante tres meses charlas sobre hábitos de estudios para los jóvenes de su comunidad, aprovechándose ese espacio para invitar a los asistentes a participar en la biblioteca. Esta fue considerada la primera actividad promocional de la biblioteca, co-gestionada con la iglesia evangélica de la zona, y contribuyó mucho en dar forma a la organización del grupo promotor de esa comunidad específica. En mérito al éxito de esta iniciativa se propuso a las otras bibliotecas empezar sus actividades con un ciclo de charlas similar, pero haciendo algunas variantes en los temas, lo cual fue aceptado y facilitó la transición a la etapa de acompañamiento. 


\section{Etapa de acompañamiento}

El acompañamiento es una etapa importante en la formación y consistió en sostener reuniones con los promotores de cada biblioteca para brindarles asesoría y coordinar actividades futuras además para absolver las dudas que iban produciéndose sobre el proyecto. En esta etapa se continuó con el enfoque constructivista pero incorporándose pautas para el trabajo con grupos pequeños de promotores (Frings, Gachuchi, Matiru \& Müller, 1993); en este sentido, en esta etapa se reforzó la característica de centrarse en la aplicación directa de lo aprendido y de aprender por medio de la aplicación y la experiencia.

La asesoría se centraba básicamente en apoyar el diseño de actividades y coordinar la provisión de recursos necesarios para gestionar las acciones que emprendían los promotores. Muchas de las actividades organizadas siguieron la línea del 'ciclo de charlas', pero poco a poco fueron diversificándose según las habilidades de cada equipo de promotores. Así, podemos mencionar las siguientes actividades: Lectura de cuentos para niños, Presentaciones de títeres, Apoyo en lecto-escritura y Asesoría en tareas escolares.

Conforme cada equipo iba funcionando, se incorporaban nuevos miembros que progresivamente aprendían las labores propias de la biblioteca mediante la transmisión de las experiencias de los miembros antiguos. Sobre este último punto cabe señalar que debido a que algunos promotores buscaban no compartir lo aprendido bajo la premisa de una supuesta superioridad de los 'antiguos' sobre los 'nuevos', se hizo un trabajo especial de sensibilización apelando a las líneas de vida tanto de cada miembro como de la misma comunidad, transmitiendo la idea de que la comunidad subsiste por la continuidad que cada generación da a las instituciones, de modo que si bien todos hemos sido 'nuevos' en algún momento, fue gracias al apoyo de generaciones pasadas que logramos incluirnos en nuestra actual comunidad.

Al momento del fin de la consultoría que tuvo como objeto la formación de promotores, se tenía a los cuatro equipos de promotores realizando actividades en forma sostenida con lo que se estimó una evaluación favorable del trabajo.

\section{CONCLUSIONES}

En esta sección se presentan las conclusiones y recomendaciones del estudio presentado, a modo de «lecciones aprendidas». A diferencia de otras formas de construcción de conocimiento, la sistematización de experiencias permite "extraer lecciones concretas aprendidas de la práctica" (Pérez, 1999: 27). Para la mejor comprensión de las lecciones aprendidas, estas son antecedidas por una reflexión de lo acontecido. Cabe reiterar que una sistematización consiste en una reconstrucción de los hechos en un marco específico y no conlleva conclusiones 'generales'; por ello, si bien las lecciones aprendidas se redactan en modo determinante, estas no deben ser tomadas como pautas con un efecto causal asegurado, tan sólo son referencias a lo que funcionó y no funcionó en la experiencia particular que ha sido objeto de este reporte. 


\section{Etapa de planificación}

1. Hacer una convocatoria amplia buscando un equilibrio de homogeneidad y diversidad entre los participantes. La homogeneidad debe establecerse según criterios etáreos, educativos y geográficos, entre otros. La diversidad debe permitir establecer relaciones de cooperación y aprendizaje intra-grupo para reforzar el buen funcionamiento de las actividades que han sido diseñadas bajo el enfoque pedagógico constructivista. En el caso de la presente experiencia, hubo homogeneidad en cuanto a la edad y la diversidad se dio por el nivel educativo, género y creencias religiosas. Esto representó una oportunidad de éxito para las sesiones ya que las diferentes opiniones enriquecían la aplicación de los aprendizajes y la relación entre pares.

2. La convocatoria debe tener como propósito llegar a personas interesadas a partir de miembros de la comunidad, obteniéndose de los involucrados una participación activa, es decir que lleve a los pobladores a asumir funciones, roles y competencias en una gestión compartida del proyecto. En muchas comunidades ocurrió que algunos pobladores asistieron a las capacitaciones y reuniones de coordinación pero a la vez se involucraban en muchos otros espacios sin desarrollar una participación consistente. En los proyectos sociales suelen presentarse estos casos protagonizados por vecinos que buscan involucrarse en actividades pero sin asumir roles, o sea, para recibir beneficios sin hacer esfuerzo (participación receptiva) o para imponer su liderazgo local y obtener 'prestigio' sin tomar parte en el desarrollo de la experiencia (participación funcional) (Bobadilla, 2006); ambas formas de participación están alejadas del enfoque de empoderamiento que se ha pretendido dar al proyecto de bibliotecas comunales.

3. Basar la convocatoria en los logros parciales que el proyecto vaya logrando. Al inicio del proceso se contaba con aliados en las comunidades, principalmente organizaciones sociales de base, pero algunos líderes no mostraron compromiso y con esto afectaron la ejecución de los talleres de capacitación; específicamente el problema fue el poco número de participantes que habían convocado. Esto se fue superando en la medida que el proyecto mostraba logros que motivaban a otros jóvenes a involucrarse. Siguiendo a Gudynas \& Evia (1994) podríamos inferir que conforme la experiencia muestra resultados tangibles (sin importar su envergadura) se promueve en las comunidades que las personas motivadas se involucren en la experiencia con un sentido de 'participación profunda', es decir, que encuentren en las actividades del proyecto la posibilidad de promover los cambios que deseen en los ambientes de su comunidad; estableciendo negociaciones con las otras personas que también persiguen promover su visión del desarrollo comunitario en el proyecto.

4. Las actividades deben programarse respetando los calendarios de cada comunidad. Así, antes de una adaptación de la comunidad al proyecto lo será del proyecto a la comunidad: es muy importante comprender el ritmo de vida de cada comunidad. En una oportunidad se programó una capacitación en la misma fecha en que se realizaba un certamen de belleza tradicional en una localidad participante; el resultado fue que no hubo más que dos asistentes a la sesión educativa. A partir de este hecho se empezó a programar actividades teniendo en consideración el calendario comunal, e 
incluso las horas de reunión fueron adaptándose de manera que los grupos adquirieran la costumbre de dedicar un día de la semana a la biblioteca.

\section{Etapa de capacitación}

1. El equipo de trabajo debe compartir criterios técnicos en cuanto al proceso de capacitación. Para alcanzar esto debe considerarse una selección científica de personal al momento de conformar el equipo y también mediante la construcción de una visión común mediante el diálogo e intercambio de experiencias en acciones similares.

2. El diseño de actividades de capacitación debe tener base en la evidencia. Aquí cobra relevancia la experiencia del equipo y de los pobladores participantes, asimismo la capacidad de innovar contenidos y de adaptar estrategias eficaces en otros ámbitos para generar nuevas aplicaciones en la propia experiencia.

3. El proceso formativo debe tener un enfoque basado en competencias. La capacitación debe contemplar el desarrollo armónico de actitudes, conocimientos y prácticas que consoliden las prácticas propuestas en el perfil de capacitación, de manera que las personas puedan realizar efectivamente el rol para el cual se están formando.

4. Las actividades de capacitación deben contemplar metodología vivencial ("aprender de uno mismo") y experiencial ("aprender haciendo"), contemplando en gran medida el componente de ensayo y acompañamiento ya que la capacitación es una práctica controlada de ensayo y error que permite lograr el aprendizaje en forma efectiva (Lara, 2001).

5. Emplear como enfoque de trabajo la perspectiva constructivista. En nuestra experiencia ha resultado valioso partir de los saberes que poseen los pobladores de comunidades para mantener constante su motivación en la actividad y alcanzar que se identifiquen con las propuestas del equipo técnico. En sí, creemos que las diversas ideas constructivistas tienen un fuerte vínculo con la gestión de los proyectos sociales en el marco del desarrollo humano ya que ambos procesos se basan fundamentalmente en el desarrollo de capacidades (Sen, 1995, 2001).

\section{Etapa de acompañamiento}

1. Basar la motivación de los promotores en el cumplimiento progresivo de expectativas ("Grandes cambios, pequeños logros"). Considerando que algunas relaciones establecidas con organizaciones que apoyaron el proyecto en un primer momento fueron debilitándose, esencialmente por el descontento surgido ante el incumplimiento de expectativas no explicitadas que muchas de ellas tenían, por ejemplo recibir computadoras o que los promotores accedan a un sueldo. La motivación de muchos líderes comunales por obtener beneficios fue quizás lo que realmente generó estas demandas ocultas, pero el valor que las bibliotecas fueron cobrando progresivamente en las localidades sirvió como motivo para que otros miembros de la comunidad dieran sostenibilidad a la experiencia ante el alejamiento de los líderes 'defraudados'. De 
esta forma se pudo establecer un vínculo más estable con la comunidad para enfrentar el reto de articular esfuerzos y difundir la experiencia.

2. Continuar la incorporación de promotores. Es importante que la experiencia no resulte un espacio 'cerrado' en la comunidad, más bien debe constituirse como una 'vía de oportunidad' para que cualquier vecino interesado en emprender actividades comunales pueda contar con una plataforma de apoyo. De esta forma los promotores comunitarios representarían un incremento en el capital social de su localidad.

3. Atender el cumplimiento de resultados (objetivos). En aras de una adecuada inversión de tiempo y energías tanto del equipo como de las comunidades, es necesario respetar los cronogramas elaborados. Estos instrumentos de planificación deben confeccionarse del modo más consensuado posible y respetando las actividades de la comunidad (como se señaló en las lecciones aprendidas de la etapa de planificación), pero además deben ser respetados para alcanzar productos en el tiempo previsto y mantener estable la motivación de los actores involucrados.

4. Brindar retroalimentación (feedback). En la experiencia fue muy relevante motivar al grupo para que estimule sus capacidades, destacando los promotores que mostraron un crecimiento de su internalidad, lo que resultó relevante para el proyecto, ya que las personas que la manifestaron asumieron posiciones de liderazgo y manifiestan su creencia en poder modificar su medio, asimismo cumplieron compromisos y fueron persistentes, consistentemente con lo referido por otros autores (Ver Bonilla, Hernández \& Camacho, 1994).

\section{REFERENCIAS BIBLIOGRÁFICAS}

1. Agencia Suiza para el Desarrollo y la Cooperación (COSUDE) (2000). Política de desarrollo social de COSUDE. En: Respecto a la dificultad de erradicar la pobreza en el mundo (pp. 14-60). Suiza.

2. Agencia Suiza para el Desarrollo y la Cooperación (COSUDE) (2002). Apoyar a América Latina para reducir la pobreza. La cooperación al desarrollo de Suiza en América Latina: Estrategia de COSUDE a mediano plazo 2002-2010 (Folleto). Suiza.

3. Altobelli, L. (2002). Participación comunitaria en la salud: La experiencia peruana en los CLAS. En: J. Arroyo (ed.). La salud peruana en el siglo XXI. Retos y propuestas de política (pp. 303-354). Lima: Consorcio de Investigación Económica y Social (CIES)

4. Avendaño, C. (2000). Aspectos psicosociales de la pobreza. En VI Seminario Internacional. Psicología de la Salud. Lima: Universidad San Martín de Porres.

5. Banister, P.; Burman, E.; Parker, I.; Taylor, M. \& Tindall, C. (1994). Qualitative Methods in Psychology. A research guide. England: Open University Press.

6. Barnechea, M. M.; González, E. \& Morgan, M de la L. (1998). La producción de conocimientos en sistematización. Ponencia en el Seminario Latinoamericano de Sistematización de Prácticas de Animación Sociocultural y Participación Ciudadana en América Latina. Medellín, Colombia, 11 al 14 de agosto. 
7. Bobadilla, P. (2006, junio). Diseño estratégico de proyectos sociales. VIII Diploma de Especialización en Diseño y Gestión de Proyectos Sociales. Pontificia Universidad Católica del Perú, Lima-Perú.

8. Bonilla, M. P.; Hernández R., A. M. \& Camacho V., M. (1994). Locus de control en adolescentes y la escolaridad de los padres. Psicología Contemporánea, 1(2), 58-61.

9. Frings, A.; Gachuchi, D.; Matiru, B. \& Müller, J. (1993). Join us in a participatory approach to training, learning \& production. A practical guide to the action training model. Germany: DSE.

10. González, R. (1998). El constructivismo. Sus fundamentos y aplicación educativa. Lima: Centro Psicosocial de Desarrollo Humano (CEDHUM).

11. Gudynas, E. \& Evia, G. (1994). Ecología social. Manual de metodologías para educadores populares. Lima: Tarea.

12. Jara, O. (1994). Para sistematizar experiencias: Una propuesta teórica y práctica. Lima: Tarea.

13. Lara, A. (2001). Manual para el trabajo comunitario. 2. ${ }^{\text {a }}$ ed. Lima: CEDEIS.

14. Loli, A. \& Paredes, E. (1999). Participación comunal en la solución de problemas psicosociales. Lima: Universidad Nacional Mayor de San Marcos.

15. Lozano, J. \& Palacios, H. (2005). Mujeres y medio ambiente: retos y posibilidades desde los Comités de Saneamiento Ambiental en Nuevo Pachacutec. En: Las relaciones entre población y medio ambiente: cuatro casos de estudio (pp. 75-105). Lima: Asociación Peruana de Demografía y Población (APDP) y Fondo de Población de las Naciones Unidas (UNFPA).

16. Ministerio de Educación (2005, noviembre 13). ¿Cuánto aprenden sus hijos en la escuela? Resultados de la Evaluación Nacional 2004. Suplemento contratado de distribución nacional. También disponible en http://www.minedu.gob.pe/umc

17. Organización para la Cooperación y el Desarrollo Económico (OCDE) y UNESCO (2003). Aptitudes básicas para el mundo de mañana - Otros resultados del proyecto PISA 2000. Resumen Ejecutivo. Canadá: OCDE-UNESCO.

18. Pérez, G. (1999). Elaboración de proyectos sociales. Madrid: Narcea.

19. Sánchez Vidal, A. (2002). Psicología social aplicada. España: Prentice Hall.

20. Sen, A. (1995). Nuevo examen de la desigualdad. Madrid: Alianza Editorial.

21. Sen, A. (2001). Desarrollo y libertad. Colombia: Planeta.

22. Tarazona, D. (2004). Acercamiento a la experiencia subjetiva del voluntariado social en organizaciones juveniles. Revista Dispersión, N. ${ }^{\circ} 3$.

23. Tarazona, D. (2006). Autoestima, satisfacción con la vida y condiciones de habitabilidad en adolescentes según pobreza y sexo. Ponencia en el $7^{\circ}$ Congreso Virtual de Psiquiatría, Mesa Temática “Actualización en trastornos infantiles y de 
la adolescencia". Disponible en http://www.psiquiatria.com/articulos/trastornosinfantiles $/ 24773$

24. Undurraga, C. \& Avendaño, C. (1998). Dimensión psicológica de la pobreza. Psykhe, 6(1), 57-63. 